University of Wollongong

Research Online

Faculty of Engineering and Information

Faculty of Engineering and Information

Sciences - Papers: Part A

Sciences

$1-1-2015$

Explosive breaching of walls with contact charges: theory and applications

Alex Remennikov

University of Wollongong, alexrem@uow.edu.au

Igor Mentus

Kamenetz-Podolsk National Technical University

Brian Uy

University of New South Wales, brianuy@uow.edu.au

Follow this and additional works at: https://ro.uow.edu.au/eispapers

Part of the Engineering Commons, and the Science and Technology Studies Commons

Research Online is the open access institutional repository for the University of Wollongong. For further information contact the UOW Library: research-pubs@uow.edu.au 


\title{
Explosive breaching of walls with contact charges: theory and applications
}

\author{
Abstract \\ Breaching activities are required for military operations in urban environment and for firefighters in \\ emergency situations for saving lives. Explosive wall breaching is a key capability in military operations in \\ urban terrain environments where the close proximity of urban structures significantly restricts the use of \\ large demolition charges. Explosive breaching is also used by special operations groups as a means to \\ gain entry into a structure where conventional breaching methods are not sufficient or the emergency \\ situation requires immediate entry to save lives. This paper develops an analytical model for the explosive \\ breaching of protective targets such as concrete and masonry walls. It provides simple analytical models \\ based on the principles of blast physics and the conservation laws for the characteristics of contact \\ charges required to produce a wall breach of the required shape and size. The model is validated using \\ the experimental data. An illustrative example is included to demonstrate the model's ability to predict the \\ size of a breach hole in a thick masonry wall. \\ Disciplines \\ Engineering | Science and Technology Studies

\section{Publication Details} \\ Remennikov, A., Mentus, I. \& Uy, B. (2015). Explosive breaching of walls with contact charges: Theory and \\ applications. International Journal of Protective Structures, 6 (4), 629-647. International Journal of \\ Protective Structures
}

This journal article is available at Research Online: https://ro.uow.edu.au/eispapers/5176 


\title{
Explosive Breaching of Walls with Contact Charges: Theory and Applications
}

\author{
Alex Remennikov', *, Igor Mentus ${ }^{2}$ and Brian Uy ${ }^{3}$ \\ ${ }^{1}$ Centre for Infrastructure Protection \& Mining Safety, University of \\ Wollongong, Wollongong, NSW, 2522, Australia \\ ${ }^{2}$ School of Military Engineering, Kamenetz-Podolsk National Technical \\ University, Kamenetz-Podolsk, Ukraine \\ ${ }^{3}$ Centre for Infrastructure Engineering, School of Civil and \\ Environmental Engineering, The University of New South Wales, \\ NSW, Australia
}

Received on 11 June 2015; Accepted 8 September 2015

\begin{abstract}
Breaching activities are required for military operations in urban environment and for firefighters in emergency situations for saving lives. Explosive wall breaching is a key capability in military operations in urban terrain environments where the close proximity of urban structures significantly restricts the use of large demolition charges. Explosive breaching is also used by special operations groups as a means to gain entry into a structure where conventional breaching methods are not sufficient or the emergency situation requires immediate entry to save lives. This paper develops an analytical model for the explosive breaching of protective targets such as concrete and masonry walls. It provides simple analytical models based on the principles of blast physics and the conservation laws for the characteristics of contact charges required to produce a wall breach of the required shape and size. The model is validated using the experimental data. An illustrative example is included to demonstrate the model's ability to predict the size of a breach hole in a thick masonry wall.
\end{abstract}

Key words: demolition, breaching failure, contact charge, explosion

\section{INTRODUCTION}

Breaching activities are required for military operations in urban environment and for firefighters in emergency situations for saving lives. Explosive wall breaching is a key capability in military operations in urban terrain environments where the close proximity of urban structures significantly restricts the use of large demolition charges. Because of this requirement, there is the need to optimise breaching activities in urban terrain. Explosive breaching is also used by special operations groups as a means to gain entry into a structure

*Corresponding author. E-mail address: alexrem@uow.edu.au 
where conventional breaching methods are not sufficient or the emergency situation requires immediate entry to save lives.

Breaching charges can be used to destroy bridge piers, bridge abutments, and for producing breaches in concrete or masonry walls during civil demolition and rescue operations. The size, shape, placement, and confinement of breaching charges are critical to successful outcome of the operation. The intent of breaching charges is to transmit enough energy to the target to create spalling and make a crater. Research related to experimental and theoretical analysis of explosive demolition of structures is very limited. Fujikake and Aemlaor [1] investigated the damage of reinforced concrete columns subjected to blasting demolition techniques and the effect of the longitudinal and shear reinforcement. Their test results confirmed that the shear reinforcement could significantly affect the damage of the columns by the demolition blasting.

Akers et al. [2] investigated new explosive wall-breaching systems and numerical techniques to model the breaching systems' interactions with the wall targets. Simple arrangements of Composition C-4 (C4) explosive were used to conduct experimental study of breaching effectiveness against reinforced concrete walls. In the numerical simulations, the concrete and reinforcing steel were modelled as Lagrangian materials, and the $\mathrm{C} 4$ was modelled as an Eulerian material. The concrete was modelled with the Microplane model. The Lagrangian meshes for the concrete walls required 1.6 to 3.5 million elements; the Eulerian meshes were typically 1.2 to 3 million cells. A typical run used 64 to 256 processors and required 48 to 12 hours to obtain 6 to 10 milliseconds of simulation time [2].

Prediction methods for damage of concrete structures caused by close-in detonations and contact charges have been proposed by a number of researchers. McVay [3] conducted 40 tests on reinforced concrete walls to investigate parameters which affect spall and to develop prediction method. The experimental program included 6 tests with $1.0 \mathrm{lb} \mathrm{C}$ 4 contact charges, but none of the tests produced a wall breach type of damage. Morishita et al. $[4,5]$ carried out a series of contact explosion tests to investigate damage of reinforced concrete slabs and effect of concrete strength and reinforcement ratio. The important findings of this study were that the concrete strength and the reinforcement spacing did not affect cratering and spalling significantly. Morishita et al. [4] also developed predictive formulas for breaching failure mode of concrete slabs using approximation of the experimental results. Beppu et al. [6] investigated failure modes of concrete slabs strengthened with FRP laminates when subjected to contact charge detonations. Test results were compared with the model formulated by Morishita et al. [4].

Riedel et al. [7] compared hydrocode simulations and the engineering tools for damage analysis of concrete structures. Gebbeken et al. [8] developed the empirical analysis tool XPLOSIM for concrete breaching predictions that uses fit functions for crater and spall area dimensions for contact and very close detonations. Since XPLOSIM methodology is not physics-based, the quality of predictions would depend on the quality of approximating functions developed using a limited number of experiments.

In some cases, the wall breach should be of a sufficient size to allow access through the wall to firefighters and members of special operation teams in emergency situations. So far, no models have been able to analytically predict the dimensions of a wall breach for contact charges. This paper presents analytical models based on the principles of blast physics and the conservation laws for the parameters of contact charges required to produce a wall breach of the required shape and size. It initially introduces the theoretical background for predicting the blast load due to contact detonation. Then, a mathematical model of wall breaching with concentrated contact charges is presented and validated against existing experimental data. 


\section{EXPLOSIVE LOADING FROM CONTACT CHARGES}

\subsection{HYPOTHESIS OF INSTANTANEOUS DETONATION}

When analysing mechanical effects of explosion, as a first approximation, it is possible not to take into consideration the detonation velocity of the explosive charge, i.e. to make an assumption that the process of explosive charge detonation is instantaneous.

For explosion in air, the air pressure is insignificant compared to the detonation pressure, as such it is possible to neglect the pressure of the surrounding air at the initial phase of expansion of detonation products. For very close distances from the explosive charge (10-15 radii of the charge), the density of the surrounding medium may also be disregarded since its value is $700-800$ times lower than that of the density of the explosive material. For more distant explosions, the density of air will be of higher importance since its density will be comparable to the density of expanded disturbed field of detonation product gases.

The main simplifying assumptions that are used to derive the analytical expressions of explosive loading on structural elements for close-range explosions can be summarized as follows: (1) detonation process is instantaneous (i.e. assume detonation velocity $D \rightarrow \infty$ ); (2) the pressure $p_{1}$ of the surrounding medium is insignificant compared to the pressure of the detonation product gases and may be neglected (i.e. assume $p_{1} \rightarrow 0$ ); and (3) the density $\rho_{1}$ of the surrounding medium is very small compared to the density of the detonation product gases (i.e. assume $\rho_{1} \rightarrow 0$ ).

According to the last two assumptions $\left(p_{1} \rightarrow 0, \rho_{1} \rightarrow 0\right)$, the surrounding medium is not present and only the detonation product gases contribute to the one-dimensional flows in the disturbed region surrounding the explosive charge. The proposed formulas shall be used with a clear understanding of the made assumptions. If the problem formulation demands conditions that are significantly different from the simplifying assumptions, other more precise (but more demanding) analytical or computational techniques should be used.

Under the condition of instantaneous detonation, all explosive charge particles are stationary because initially they hold the volume of the original explosive. After the instantaneous explosion, the detonation product gases begin to expand. The particles located on the outer surface of the charge begin flying away first. Following the outer surface particles, the particles located on the successive interior surfaces start progressively flying away so that the boundary between the moving particles and the stationary particles is moving inside the charge with some velocity. This moving boundary can be termed the hypothetical surface of the expanding detonation products. It is noteworthy that in the real process of detonation the disturbances move back inside the detonation products with the velocity of sound in the detonation products, and the boundary of this disturbance is the head of the rarefaction wave after which the explosion parameters vary smoothly. The assumptions of an instantaneous detonation and the hypothetical surface for the explosive products provide sufficiently accurate solution for the blast parameters in the surrounding medium only for the region within 10-15 charge radii from the charge. Figure 1 shows a general picture of the idealized expansion of the detonation products.

\subsection{IMPULSE LOADING DUE TO CONTACT EXPLOSION}

An explosive charge that is detonated in contact with an obstacle can be represented as a volume filled with condensed (solid or liquid) matter. The principal characteristics of an explosive charge are: explosive mass, $C$ (kg TNT); specific energy of detonation, $Q_{0}$ $(\mathrm{MJ} / \mathrm{kg})$; density of explosive, $\rho_{0}\left(\mathrm{~kg} / \mathrm{m}^{3}\right)$; and detonation velocity, $D_{0}(\mathrm{~m} / \mathrm{sec})$.

Detonation of a high explosive is complex phenomenon. An initial shock compresses a high-explosive material, heating it and causing chemical decomposition. The formation of 

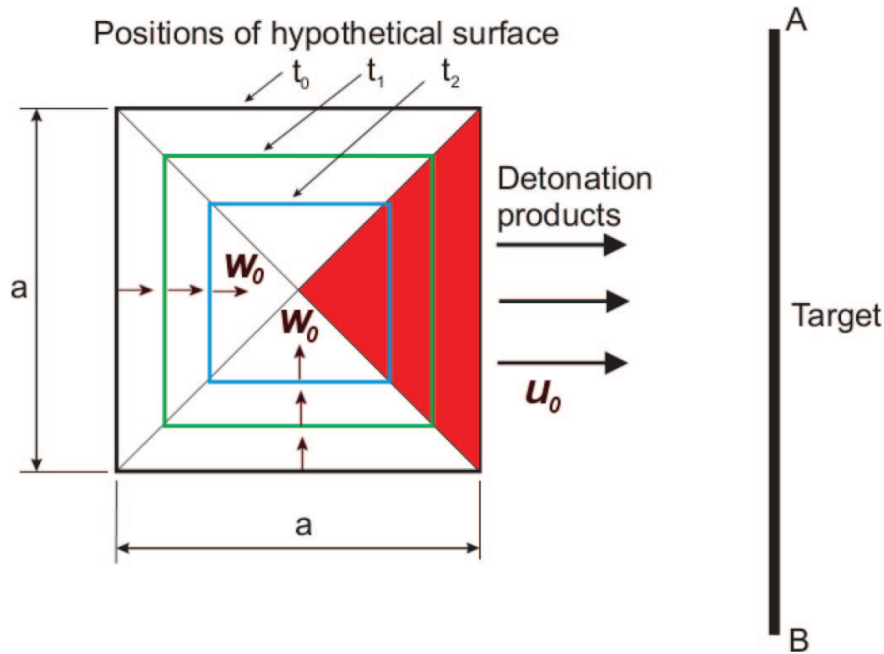

Figure 1. Hypothetical surface for expanding detonation products for cubic HE charge

chemical products releases enormous amount of energy in just millionth of a second. All of this happens almost instantaneously, e.g. typically detonation velocity is about $6 \mathrm{~km} / \mathrm{sec}$, to produce a blast of rapidly expanding hot gases. To determine the parameters of explosive loading, a system of differential equation describing conservation laws [10,11] is solved using numerical integration techniques and specialized computer programs.

To develop simplified engineering-level relationships for explosive loads, it is hypothesized that detonation is an instantaneous process where the volume, initially occupied by the condensed matter, is filled instantaneously by hot, highly compressed detonation product gases. The mean detonation pressure within the charge can be determined as

$$
p_{0}=\frac{\rho_{0} D_{0}^{2}}{2(k+1)}
$$

where $k$ is the polytropic exponent of the detonation products. The polytropic exponent typically used is $k \approx 3$. The exact value of $k$ lies within the range $2.54 \leq k \leq 3$, depending on the kind of explosive.

\subsection{BLAST PRESSURE ON THE TARGET AND ITS DURATION FROM CONTACT CHARGES}

Detonation product gases can only expand from the free surfaces of the charge. If the charge is in contact with the target on some of its faces, the pressure at all contact points of the target will be the same and equal to the mean detonation pressure $p_{0}$ (see eqn (1)) acting over some period of time $\tau$. Apparently, this pressure $p_{0}$ will stay unchanged until after the rarefaction wave reaches the point of interest on the target. Adopting a hypothesis of an instantaneous detonation, we will substitute the rarefaction wave with the hypothetical surface for the detonation as illustrated in Figure 1. The velocity of the hypothetical surface, $w_{0}$ is constant for a given explosive material. Hence, the duration of blast loading acting on a fixed point of the target at the contact surface between the charge and the target can be determined using the following simple relationship 


$$
\tau=\frac{\chi}{w_{0}}
$$

where $\chi$ is the distance from the point of interest on the target to the nearest free hypothetical surface, and $w_{0}$ is the velocity of the hypothetical rarefaction surface.

Thereby, all points of the target in contact with the charge will be subject to the same pressure $p_{0}$ but the duration of loading will be different for different points; the further away the point lies from the free surface of the charge, the longer time of arrival of the hypothetical surface will be. In other words, the hypothetical surface has to travel longer distance from the closest free surface of the charge in order to reach this point and relief the pressure.

Figure 2(a) shows a prismatic charge in contact with the target at its bottom surface. Points $A, B$ and $C$ will experience same pressure, but the time of arrival of the hypothetical surface at these points and consequently the time of action of the pressure will be different. The hypothetical surface reaches point $A$ at the time of detonation, hence the pressure duration is zero at point $A$. The hypothetical surface from the nearest free edge of the charge will travel distance $A B$ to reach point $B$. The duration of pressure at point $B$ will be

$$
\tau_{B}=\frac{\chi_{B}}{w_{0}}=\frac{A B}{w_{0}} .
$$

The hypothetical gas expansion surfaces from the side face and from the top face will reach point $C$ at the same time. For point $C$,

$$
\tau_{C}=\frac{\chi_{C}}{w_{0}}=\frac{A C}{w_{0}} .
$$

Figure 2(b) depicts the pressure variation with time for points $B$ and $C$.

\subsection{SPECIFIC IMPULSE ON THE TARGET FROM CONTACT CHARGE}

The duration of loading of the real obstacles by the detonation products is very short compared to the time required to displace the targets. Therefore, it is possible to assume that

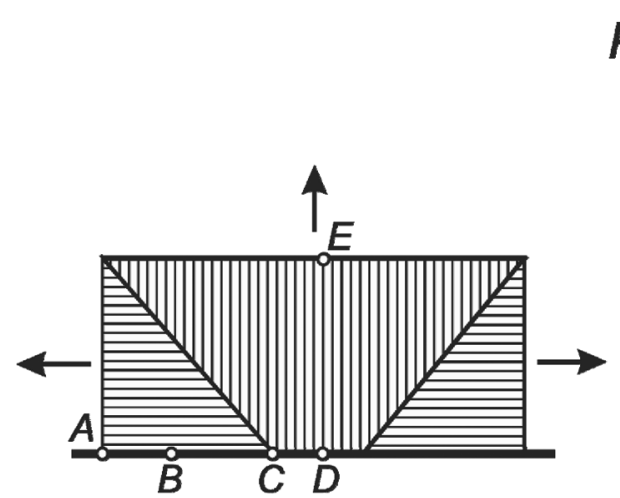

(a)

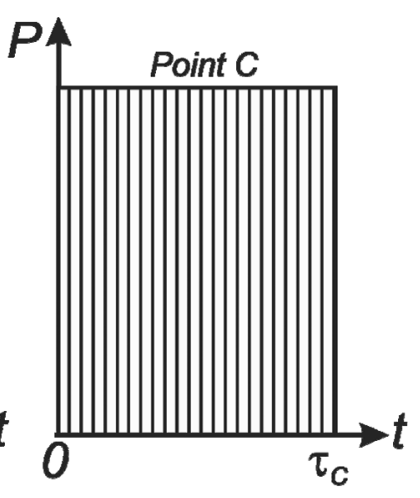

(b)

Figure 2. (a) Diagram showing directions for expanding detonation products for contact explosion; and (b) pressure variation with time for points $B$ and $C$ 
the target remains stationary and only acquires an initial velocity. The blast loading in this case can be characterized by the specific impulse

$$
i=\int_{0}^{\tau} p d t
$$

In case of the contact detonation, the pressure acting on a target remains practically constant during the load duration $\tau$, thus

$$
i=p_{0} \tau
$$

Substituting the pressure duration $\tau$ with its value from eqn (2), we get

$$
i=p_{0} \frac{\chi}{w_{0}}
$$

Using a hypothesis of instantaneous detonation and the law of conservation of momentum, it can be shown that the velocity of the hypothetical surface $[10,11]$ can be determined as

$$
w_{0}=\frac{p_{0}}{\rho_{0} u_{0}}
$$

where $u_{0}$ is the velocity of the particles of the expanding detonation products. The particle velocity of detonation products can be determined using the energy balance assuming that full explosive energy, $Q_{0}$, initially existing within the charge volume with pressure $p_{0}$ as the potential energy is transformed into the kinetic energy of the outflowing detonation products as follows

$$
u_{0}=\sqrt{2 Q_{0}}
$$

Thus, eliminating $p_{0}$ from eqn (7), we can write

$$
i=\chi \rho_{0} u_{0}
$$

where $u_{0}$ is determined from eqn (9). Eqn (10) determines the distribution of the specific impulse over the target at the points of contact with the charge. Recall that $\chi$ in eqn (10) represents a distance from the point where the blast load needs to be determined to the nearest free surface of the charge.

Figure 3 illustrates the specific impulse distributions on the target surface in contact with a cylindrical or prismatic charge with a diameter (width) $b$ and a height of $H$. For both cases $b>2 H$ and $b<2 H$, we first bisect the free corners to form the outburst areas.

Three segments $A B, B B^{\prime}$, and $B^{\prime} A^{\prime}$ can be identified along the contact surface in Figure 3(a). The hypothetical rarefaction surface from the top free face of the charge can only reach the central segment $B B^{\prime}$, therefore for all points on $B B^{\prime}$ the distance $\chi=H$. For the segments $A B$ and $B^{\prime} A^{\prime}$, the hypothetical rarefaction surface approaches from the free side faces of the charge: for points $A$ and $A^{\prime} \chi=0$, for points $B$ and $B^{\prime} \chi=H$. It can be seen that the distance $\chi$ increases from point $A$ to point $B$ and from point $A^{\prime}$ to point $B$ '. Thus, a meridional section of the specific impulse diagram takes the shape as shown in Figure 3(a). In a threedimensional space, the diagram is a truncated cone with a height equal to the maximum specific impulse $i_{\max }=H \rho_{0} u_{0}$, with the bottom base diameter being equal to the diameter of the charge, and the top base diameter being equal to $b-2 H$. 


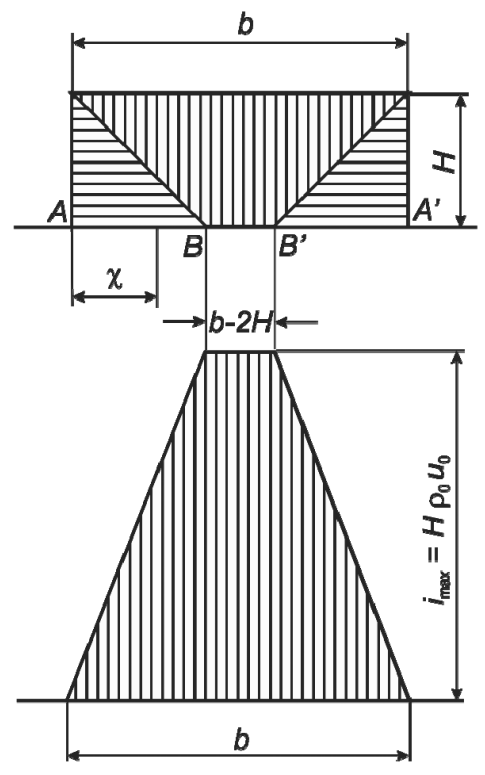

(a)

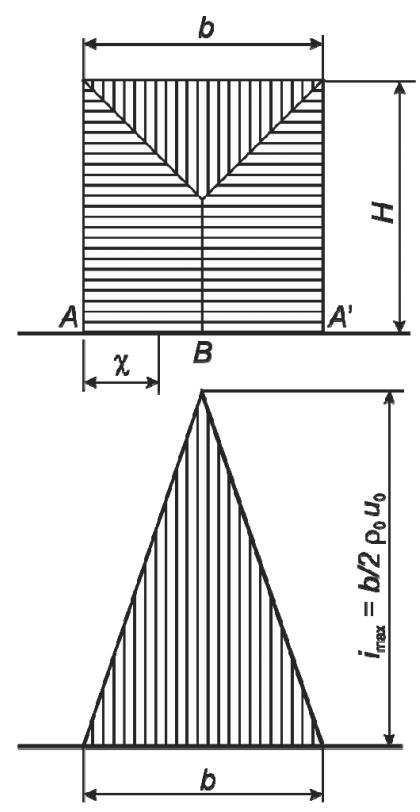

(b)

Figure 3. Specific impulse distribution for a cylindrical charge: (a) $b \geq 2 \mathrm{H}$; (b) $b \leq 2 H$

For a cylindrical or prismatic charge with $b<2 H$, the bisecting lines at the free corners of the charge form the outburst areas as shown in Figure 3(b). The most loaded point of the target $B$ (under centre of the charge) is located at a distance $\chi=b / 2$ from the nearest (side) face of the charge. Thus, at point $B$ the specific impulse

$$
i=i_{\max }=\frac{b}{2} \rho_{0} u_{0}
$$

For all other points on the contact surface, the specific impulse linearly diminishes with a distance. The meridional section of the specific impulse diagram is shown in Figure 3(b). In a three-dimensional space, this diagram is a cone with a height equal to the maximum specific impulse $i_{\max }$, and a base diameter equal to the diameter of the charge.

\subsection{SPECIFIC IMPULSE ON THE TARGET FROM CONTACT CHARGE}

The total impulse $I$ acting on the target from a contact explosion is equal to the volume of the three-dimensional diagram of the specific impulse. Thus, for a charge of cylindrical shape as depicted in Figure 3(a) the total impulse $I$ can be determined as follows

$$
\begin{aligned}
I= & \frac{1}{3} H \rho_{0} u_{0}\left(\frac{\pi b^{2}}{4}+\frac{\pi(b-2 H)^{2}}{4}+\frac{\pi}{4} \sqrt{b^{2}(b-2 H)^{2}}\right) \\
& -\frac{1}{3} \frac{\pi}{4} H \rho_{0} u_{0}\left(3 b^{2}-6 b H+4 H^{2}\right) \\
= & \frac{\pi b^{2}}{4} H \rho_{0} u_{0}\left(1-2 \frac{H}{b}+\frac{4}{3} \frac{H^{2}}{b^{2}}\right) .
\end{aligned}
$$


Since the charge mass $C$ can be determined as

$$
C=\frac{\pi b^{2}}{4} H \rho_{0}
$$

then, introducing the parameter in eqn (12)

$$
\mu=1-2 \frac{H}{b}+\frac{4}{3} \frac{H^{2}}{b^{2}}
$$

we get the following design formula for the total impulse of a cylindrical charge with $b \geq 2 \mathrm{H}$

$$
I=u_{0} C \mu
$$

The parameter $\mu$ can be termed a charge shape coefficient as it depends only on the charge linear dimensions and can be determined for the charges of different shapes such as prismatic, cylindrical, and hemispherical contact charges placed with their base on the target. For instance, the total impulse of a cylindrical charge with its base in contact with the target is determined by eqn (15) with the charge shape coefficient

$$
\mu=\left\{\begin{array}{l}
1-2 \frac{H}{b}+\frac{4}{3} \frac{H^{2}}{b^{2}}, \quad \text { if } \frac{b}{H} \geq 2 ; \\
\frac{b}{6 H}, \quad \text { if } \frac{b}{H} \leq 2
\end{array}\right.
$$

For a prismatic charge with the dimensions $b$ and $l(l \geq b)$ and height $H$, the charge shape coefficient can be determined using a similar approach to eqns (13-15) as follows

$$
\mu=\left\{\begin{array}{l}
1-\frac{H}{b}-\frac{H}{l}+\frac{4}{3} \frac{H^{2}}{b l}, \quad \text { if } \frac{b}{H} \geq 2 ; \\
\frac{1}{4} \frac{b^{2}}{l H}\left(\frac{l}{b}-\frac{1}{3}\right), \quad \text { if } \frac{b}{H} \leq 2 .
\end{array}\right.
$$

Eqns (16-17) can be used to determine the total impulse for flat high-explosive charges, e.g. sheet explosive charges. For such charges, $H / b \ll 1$. so the charge shape coefficient $\mu=1$.

Values of $\mu$ for some values of ratio $b / H$ are provided in Table 1 . One can see from Table 1 that the total impulse transferred to the target plate is significantly affected by the ratio between the width (breadth) of the charge and its height. Table 1 clearly shows that the charges with the ratio $b / H<2$ are not effective. This recommendation is adopted in the current manuals for demolition works [9].

Table 1. Values of charge shape coefficient for cylindrical charges

\begin{tabular}{cccccccccc}
$\boldsymbol{b} / \boldsymbol{H}$ & $\mathbf{1} / \mathbf{1 0}$ & $\mathbf{1 / 2}$ & $\mathbf{1}$ & $\mathbf{2}$ & $\mathbf{3}$ & $\mathbf{4}$ & $\mathbf{5}$ & $\mathbf{1 0}$ & $\mathbf{2 0}$ \\
\hline & 0.02 & 0.08 & 0.17 & 0.33 & 0.48 & 0.58 & 0.65 & 0.81 & 0.90 \\
\hline
\end{tabular}




\section{BREACHING WALLS USING CONCENTRATED CONTACT CHARGES 3.1. EMPIRICAL DESIGN RECOMMENDATIONS}

Military manual [9] provides the recommendations how to determine the size of the charge required to breach concrete or masonry walls by using the following empirical formula

$$
P=R^{3} K C
$$

$P$ is the explosive charge (pounds TNT), $R$ is the breaching radius (feet), $K$ is the material factor which reflects the strength, hardness and mass of the material, and $C$ is the tamping factor, which depends on the location and tamping of the charge.

The breaching radius $R$ for external charges should be selected equal to the thickness of the target being breached. For example, when breaching a 1-metre thick wall with an external contact charge, the breaching radius of $1.0 \mathrm{~m}$ should be selected in eqn (18). Table 2 provides recommended values of the material factor $K$ for breaching charges for concrete and masonry targets. Material factor $K$ represents the strength and hardness of the target material. When the target material cannot be positively identified, the target consisting of the strongest type of material in the group should be assumed.

The tamping factor $C$ depends on the charge location and materials used for tamping. Figure 4 illustrates some methods for placing charges and provides the values of $C$ for both tamped and untamped charges.

The existing empirical relationships similar to eqn (18) can only predict the size of the charge required to breach concrete, masonry or similar material walls. These relationships are not suitable for the prediction of the shape, dimensions and mechanism of formation of a breach opening. A new mathematical model of wall breaching presented in the next section provides a means for predicting the geometric parameters of a breach hole and constructing a graphical picture of wall breaching by contact charges.

Table 2. Material factor ( $K$ ) for breaching charges (9)

\begin{tabular}{llc} 
Material & Breaching Radius $(\boldsymbol{R})$ & Material Factor $(\boldsymbol{K})$ \\
\hline Poor masonry & Less than $1.5 \mathrm{~m}$ & 0.32 \\
& $1.5 \mathrm{~m}$ or more & 0.29 \\
Good masonry, & $0.3 \mathrm{~m}$ or less & 0.88 \\
Concrete block & Over $0.3 \mathrm{~m}$ to less than $0.9 \mathrm{~m}$ & 0.48 \\
& $0.9 \mathrm{~m}$ to less than $1.5 \mathrm{~m}$ & 0.40 \\
& $1.5 \mathrm{~m}$ to less than $2.1 \mathrm{~m}$ & 0.32 \\
& $2.1 \mathrm{~m}$ or more & 0.27 \\
Dense concrete, & $0.3 \mathrm{~m}$ or less & 1.14 \\
First-class masonry & Over 0.3 m to less than $0.9 \mathrm{~m}$ & 0.62 \\
& $0.9 \mathrm{~m}$ to less than $1.5 \mathrm{~m}$ & 0.52 \\
& $1.5 \mathrm{~m}$ to less than $2.1 \mathrm{~m}$ & 0.41 \\
Reinforced concrete & $2.1 \mathrm{~m}$ or more & 0.35 \\
(without considering cutting & $0.3 \mathrm{~m}$ or less & 1.76 \\
reinforcement) & Over 0.3 m to less than $0.9 \mathrm{~m}$ & 0.96 \\
& $0.9 \mathrm{~m}$ to less than $1.5 \mathrm{~m}$ & 0.80 \\
& $1.5 \mathrm{~m}$ to less than $2.1 \mathrm{~m}$ & 0.63 \\
& $2.1 \mathrm{~m}$ or more & 0.54 \\
\hline
\end{tabular}



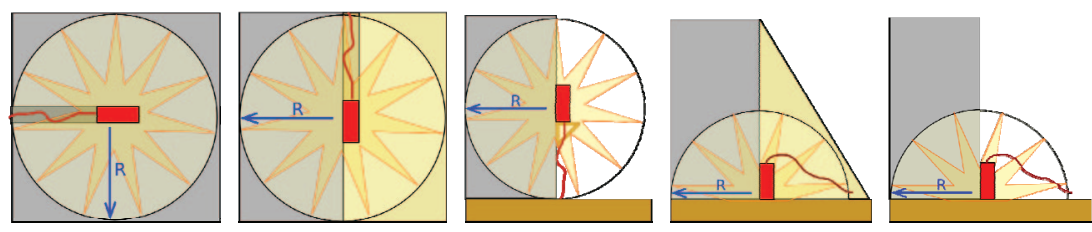

\begin{tabular}{|c|c|c|c|c|}
\hline $\begin{array}{c}C=1.0 \\
\text { Charge } \\
\text { placed in the } \\
\text { centre of the } \\
\text { mass }\end{array}$ & $\begin{array}{c}C=1.0 \\
\text { Charge is } \\
\text { temped or } \\
\text { stemmed }\end{array}$ & $\begin{array}{c}C=1.8 \\
\text { Charge is } \\
\text { elevated, } \\
\text { untamped }\end{array}$ & $\begin{array}{c}C=2.0 \\
\text { Charge is } \\
\text { ground- } \\
\text { placed, earth } \\
\text { tamping }\end{array}$ & $\begin{array}{c}C=3.6 \\
\text { Charge is } \\
\text { ground- } \\
\text { placed, } \\
\text { untamped }\end{array}$ \\
\hline
\end{tabular}

Figure 4. Tamping factor (C) for breaching charges (based on (9))

\subsection{MATHEMATICAL MODEL OF WALL BREACHING BY CONCENTRATED CONTACT CHARGES}

\subsubsection{Particle velocities of the target}

The theoretical model of the target breaching is based on the determination of particle velocities of the target under the impact loading in a form of a short-duration specific impulse $i$ acting over an area with a radius $X$ (see Figure 5). The particles of the target directly affected by the impulse loading are assumed to start displacing first. As they displace, the underlying particles and some of the sideways particles will also become involved in the displacement of the target. At the end, all particles within the truncated cone with a radius of top circle $X$ and a radius of base circle will be moving, where $\alpha$ is a halfangle at the vertex angle of the cone.

As an approximation, we can assume that the material density within the conical volume does not vary significantly during the time of involving the particle in motion. Then, using the law of conservation of mass we get

Mass flow rate in section $Z=$ Mass flow rate in section $(h+X \cot \alpha)$

Mass flow rate can be calculated by

$$
\frac{d m}{d t}=\rho u A
$$

where $\rho$ is the mass density of the target material, $u$ is the particle velocity, and $A$ is the crosssectional area required to calculate the mass flow rate. Using Figure 5 and equating the mass flow rate in the section $Z$ to the mass flow rate in the section on the surface opposite to the charge location, we get the following relationship

$$
\rho \pi(Z \tan \alpha)^{2} \cdot u=\rho \pi(h \tan \alpha+X)^{2} \cdot u_{\text {surf }}
$$

From eqn (20), we can find the particle velocity as

$$
u=u_{\text {surf }}\left(\frac{h \tan \alpha+X}{Z \tan \alpha}\right)^{2}
$$

Eqn (21) allows calculating the particle velocities of the target for the given velocity of the particles on the external surface of the same target, $u_{\text {surf }}$. 


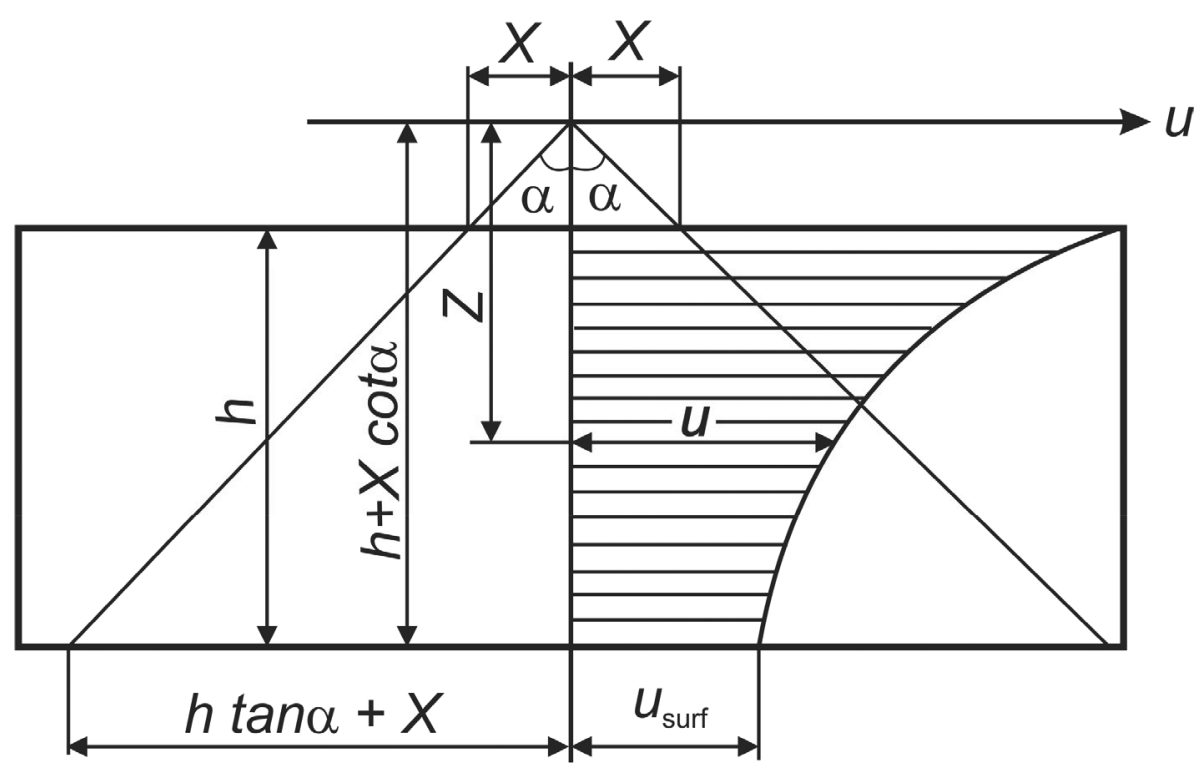

Figure 5. Diagram for the determination of particle velocity of the target subjected to uniform specific impulse $i$ over the area with radius $X$

In order to find $u_{\text {surf }}$ we can employ the law of conservation of momentum and eqn (21). According to the law of conservation of momentum, the total impulse $I$ acting on the target is equal to the change in momentum, thus

$$
\begin{aligned}
I & =\int_{m} u d m=\int_{X \cot \alpha}^{h+X \cot \alpha} u_{\text {surf }}\left(\frac{h \tan \alpha+X}{Z \tan \alpha}\right)^{2} \rho \pi Z^{2} \tan ^{2} \alpha d z \\
& =\pi \rho u_{\text {surf }}(h \tan \alpha+X)^{2} h,
\end{aligned}
$$

because $d m=\rho \pi Z^{2} \tan ^{2} \alpha d z$. From eqn (22), velocity of the particles on the external surface of the target can be determined as

$$
u_{\text {surf }}=\frac{I}{\pi \rho(h \tan \alpha+X)^{2} h}
$$

\subsubsection{Critical particle velocity}

One can make an assumption that destruction of materials is observed at the locations where the kinetic energy density induced by the blast exceeds the ultimate toughness of the material, $A_{m}$, which is the integrated area under the entire stress-strain curve to the break point. Kinetic energy density or kinetic energy per unit volume is expressed as

$$
q=\frac{\rho u^{2}}{2}
$$

Since the material mass density $\rho$ is constant for the given material the kinetic energy density is characterised only by the velocity of the particles of the target, $u$. To determine the 
critical value of the particle velocity leading to target material destruction, the kinetic energy density is equated to the ultimate material toughness $\left(\mathrm{J} / \mathrm{m}^{3}\right)$ so that

$$
\frac{\rho u_{c r}^{2}}{2}=A_{m}
$$

or

$$
u_{c r}=\sqrt{\frac{2 A_{m}}{\rho}}
$$

\subsubsection{Charge size required to breach the target}

In the limiting case, when the total energy of the particles at the free surface of the target with the acquired velocity $u_{\text {surf }}$ is expended only on the destruction of the target's material, we get

$$
u_{\text {surf }}=u_{c r}
$$

or, using eqns (23) and (26)

$$
\frac{I}{\pi \rho(h \tan \alpha+X)^{2} h}=\sqrt{\frac{2 A_{m}}{\rho}}
$$

Substituting the total impulse $I$ from eqn (15) and recalling that $u_{0}=\sqrt{2 Q_{0}}$, the following relationship for the charge size is obtained

$$
C=\pi \sqrt{\frac{\rho A_{m}}{Q_{0}}} \frac{1}{\mu}\left(\tan \alpha+\frac{x}{h}\right)^{2} h^{3}
$$

or

$$
C=K_{0} \frac{n^{2}}{\mu} h^{3}
$$

where

$$
\begin{gathered}
K_{0}=\pi \sqrt{\frac{\rho A_{m}}{Q_{0}}} \\
n=\tan \alpha+\frac{X}{h}
\end{gathered}
$$

Eqns (30-31) can be used to determine the charge size with a relationship to the spall size coefficient $(n)$, material properties of the target $\left(\rho, A_{\mathrm{m}}\right)$, properties of the explosive material $\left(Q_{0}\right)$, thickness of the target $(h)$ and charge shape ( ). Furthermore, for a given charge size eqns (30) and (31) provide a means for predicting the diameter of the spalling crater on the surface opposite to the explosive as $2 n h$. 
Parameter $K_{0}$ as follows from eqn (31), depends only upon the properties of the target material and properties of explosive. It can be interpreted as specific mass of TNT contact charges required for destruction of $1 \mathrm{~m}^{3}$ of target material. The values of $K_{0}$ can be calculated using eqn (31). Some values of the specific mass $K_{0}$ based on TNT explosive for steel, concrete and other materials are presented in Table 3.

It should be noted that the minimum radius $X$ of the area of application of the specific impulse for cylindrical and prismatic charges is required to be as

$$
\begin{cases}X=\frac{b-H}{2}, & \text { if } \frac{b}{H} \geq 2 \\ X=\frac{b}{4}, & \text { if } \frac{b}{H} \leq 2\end{cases}
$$

\subsubsection{Prediction of the size of the ejection crater}

The mathematical model for contact charge breaching of targets presented above provides more information about mechanics of target destruction than empirical formulas. For such materials as concrete, reinforced concrete, rocks, timber etc. the general form of a relationship between the charge weight, $C$ and the thickness $h$ of the member being destroyed remains the same as for metals. That is, one should expect that eqn (30) qualitatively describes the process of explosive destruction of these materials as well. However, the quantitative assessments may be significantly different due to the differences in the mechanisms of destruction of these materials and metals.

Explosion of contact charges on the steel plate results not only in the spalling crater but also in the depression and the ejection crater in the contact zone. Destruction of elements from concrete and masonry by contact charges also produces the ejection crater as well as the spalling crater. If the front-face ejection crater and the back-face spalling crater intersect, a cleared hole is produced through the wall, and the wall is considered breached as shown in Figure 6.

The radius of the ejection crater can be determined for concentrated contact charges using the following relationship $[10,11]$

$$
C=18 K r_{c}^{3}
$$

where $C$ is the required contact charge weight, $K$ is the specific charge weight for forming an ejection crater in the target material, and $r_{c}$ is the radius of the ejection crater. The physical meaning of the specific charge weight for forming an ejection crater can be explained by the following formula

\section{Table 3. Values of specific mass of explosive, $K_{0}$}

\begin{tabular}{lc} 
Material & $\boldsymbol{K}_{\mathbf{0}}\left(\mathbf{k g} / \mathbf{m}^{\mathbf{3}}\right)$ \\
\hline Steel Grade 350 & 2000 \\
High-strength steel & 2700 \\
Aluminium alloys & 1250 \\
Masonry & $5.6-9.6$ \\
Plane concrete & $12-14.5$ \\
Reinforced concrete (without cutting reinforcement) & 40 \\
Reinforced concrete (with partial cutting of reinforcement) & 160 \\
\hline
\end{tabular}




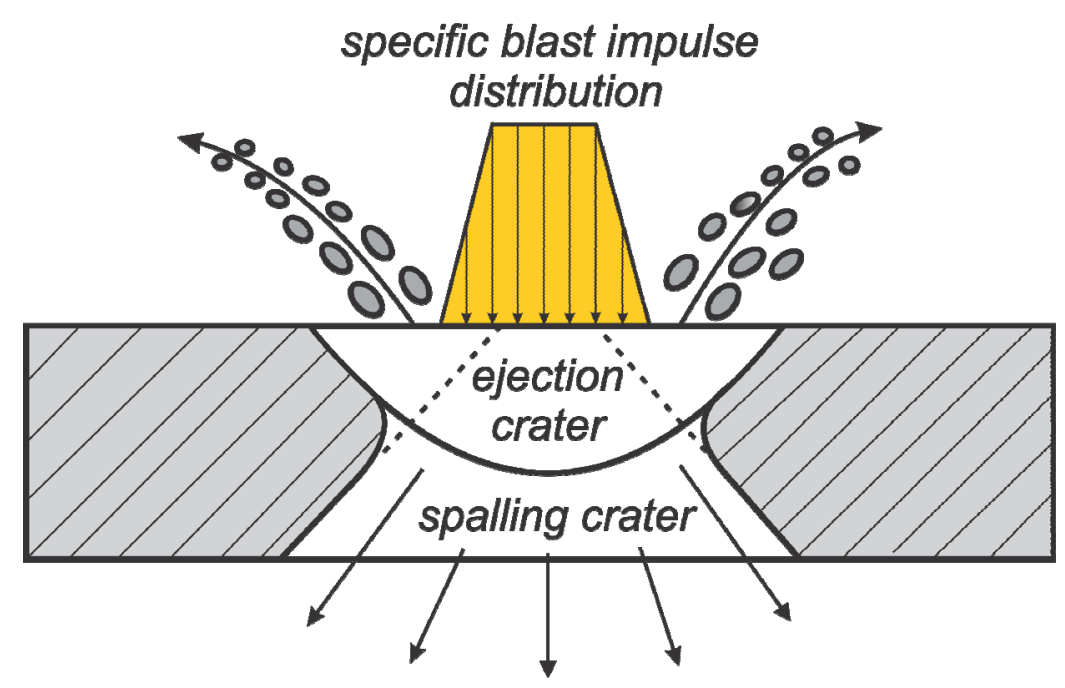

Figure 6. Mechanism of concrete breaching with contact charge

Table 4. Values of specific charge weight, $K$

\begin{tabular}{lc} 
Material & $\boldsymbol{K}\left(\mathbf{k g} / \mathbf{m}^{\mathbf{3}}\right)$ \\
\hline Masonry & $1.2-2.0$ \\
Plane concrete & $2.5-3.0$ \\
Reinforced concrete (without cutting reinforcement) & 8.4 \\
Reinforced concrete (with partial cutting of reinforcement) & 33.6 \\
\hline
\end{tabular}

$$
K=\frac{A_{M}}{Q_{0}}
$$

where $A_{m}$ is toughness of a material or the amount of energy per unit volume that a material can absorb before rupturing $\left(\mathrm{J} / \mathrm{m}^{3}\right)$, and $Q_{0}$ is the energy of explosion $(\mathrm{J} / \mathrm{kg})$.

Table 4 presents recommended values of the specific charge weight $K$ for several materials that are typically used for the wall barrier construction. These values were calculated using eqn (35) with the energy of explosion $Q_{0}$ taken for TNT explosive material $[10,11]$.

\section{VALIDATION STUDY}

In order to provide validation to the proposed analytical model for wall breaching with contact charges, the experimental data developed by Beppu et al. [6] were used. Beppu et al. tested 14 square concrete plates $500 \mathrm{~mm} \times 500 \mathrm{~mm}$ and with a thickness of $80 \mathrm{~mm}$ using contact cylindrical charges. The concrete compressive strength for all plates was 28.9 MPa. Composition C-4 (C4) explosive was used to manufacture cylindrical charges with the mass of $46 \mathrm{~g}$ and the ratio of the height to diameter 1:1. According to Beppu et al. [6], this charge mass was set to satisfy the breach threshold condition proposed by Morishita et al. [4]

$$
T / W^{1 / 3}=2.0
$$


where $T$ is the thickness of a concrete plate $(\mathrm{cm})$, and $W$ is the equivalent TNT charge mass $(\mathrm{g})$.

Cases 1 and 2 in the testing program included unreinforced concrete panels, which were used for the validation of the model presented in this paper. Figure 7 shows the failure modes of concrete panels observed in the tests. Since the charge mass was selected on a basis of causing damage at the onset of breach failure, the failure modes for cases 1 and 2 represented "crater and spall" and "breach" conditions, thus generally agreeing with Morishita's formula that the $46 \mathrm{~g} \mathrm{C} 4$ charge (57.5 g TNT equivalent) would cause the near-breach damage in the 80-mm concrete panels.

It should be noted that Beppu et al. [6] did not provide the dimensions of the cylindrical charges used in the tests. For the purposes of this validation study, the charge dimensions were calculated using a $\mathrm{C} 4$ density of $1570 \mathrm{~kg} / \mathrm{m}^{3}$ and the height to diameter ratio of 1.0 . The diameter and height of the $\mathrm{C} 4$ charges were determined to be $34 \mathrm{~mm}$.

Calculations of the breach model parameters and comparison with the experimental values are presented in Table 5 .

The results of modelling of the concrete panels subjected to a detonation of contact cylindrical charges in Table 5 demonstrate the robustness of the analytical model and good correlation with the experimental data presented by Beppu et al. in [6]. The analytical model predicted exactly the same mass of an explosive charge required for breaching the concrete panels as determined experimentally by Morishita et al. [4] and Beppu et al. [6]. The analytical model also predicted the diameter of spall, $D$, and the depth of ejection crater, $p$ that match the experimental values from [6] very closely. The ejection crate diameter, $D_{1}$, is over-predicted by $25-30 \%$ compared to the experimentally determined values, which is on the conservative side of predicting the concrete panel damage.

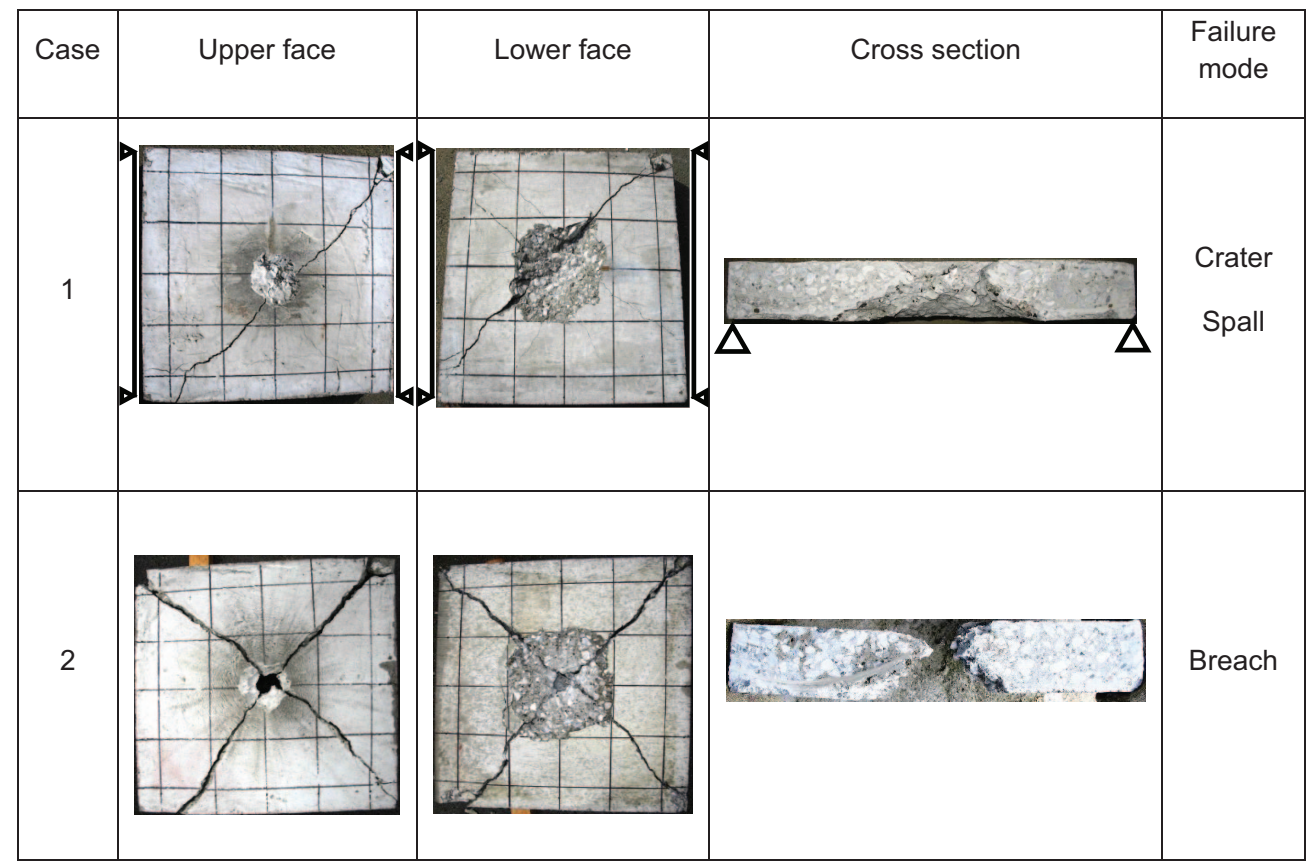

Figure 7. Failure modes of concrete panels (reproduced with permission from (6)) 
Table 5. Results of modelling response of concrete slab to contact charges

\begin{tabular}{|c|c|c|c|}
\hline Model parameter & $\begin{array}{c}\text { Reference } \\
\text { formula }\end{array}$ & $\begin{array}{l}\text { Analytical model } \\
\text { parameters and } \\
\text { predicted values }\end{array}$ & $\begin{array}{c}\text { Experimental } \\
\text { values [6] }\end{array}$ \\
\hline Concrete panel thickness, $h$ & & $80 \mathrm{~mm}$ & \\
\hline Charge mass, $C$ TNT NEQ & & $0.0575 \mathrm{~kg}$ & \\
\hline Charge height, $H$ & & $34 \mathrm{~mm}$ & \\
\hline Charge width, $b$ & & $34 \mathrm{~mm}$ & \\
\hline Charge shape coefficient, $\mu$ & Eqn (17) & 0.17 & \\
\hline $\begin{array}{l}\text { Minimum radius of area of } \\
\text { application of impulse, } X\end{array}$ & Eqn (33) & $17 \mathrm{~mm}$ & \\
\hline The spall coefficient, $n$ & Eqn (32) & 1.22 & \\
\hline $\begin{array}{l}\text { Required TNT charge to } \\
\text { cause breach }\left(K_{0}=13 \mathrm{~kg} / \mathrm{m}^{3}\right. \\
\text { Table } 3 \text { for plain concrete })\end{array}$ & Eqn (30) & $0.056 \mathrm{~kg}$ & $0.0575 \mathrm{~kg}$ \\
\hline Diameter of spalling crater, $D$ & $2 n h$ & $195 \mathrm{~mm}$ & $210 \mathrm{~mm}$ \\
\hline $\begin{array}{l}\text { Diameter of ejection crater, } \\
D_{1}\left(K=3.0 \mathrm{~kg} / \mathrm{m}^{3} \text { Table } 4\right. \\
\text { for plain concrete })\end{array}$ & Eqn (34) & $\begin{array}{l}r_{c}=102 \mathrm{~mm} \\
D_{1}=204 \mathrm{~mm}\end{array}$ & $130-160 \mathrm{~mm}$ \\
\hline Depth of ejection crater, $p$ & $0.2 r_{c}$ & $21 \mathrm{~mm}$ & $20-28 \mathrm{~mm}$ \\
\hline
\end{tabular}

\section{ILLUSTRATIVE EXAMPLE}

In an emergency situation, firefighters require gaining entry into a structure through the existing masonry wall with a thickness of $h=0.8 \mathrm{~m}$. This requires a breach hole with a minimum diameter of $1.0 \mathrm{~m}$ to be formed explosively. Determine: 1) the charge weight (TNT kg) required to form a man-passable breach hole; 2) determine the cratering parameters of the damaged wall, and 3) construct graphically the process of wall destruction.

1) To determine the size of the breaching TNT charge, we employ eqn (30)

$$
C=K_{0} \frac{n^{2}}{\mu} h^{3}
$$

Using this formula, check if one concentrated contact charge in a form of a standard $25 \mathrm{~kg}$ box of TNT explosive would be sufficient for guaranteed breaching the masonry wall. For the contact charge of a prismatic shape we get $C=25 \mathrm{~kg}, l=350 \mathrm{~mm}, b=300 \mathrm{~mm}$, and $H=150 \mathrm{~mm}$.

From Table 3, for masonry construction we take the maximum value of $K_{0}=9.6 \mathrm{~kg} / \mathrm{m}^{3}$. The charge shape coefficient is calculated according to eqn (17):

$$
\mu=1-\frac{H}{b}-\frac{H}{l}+\frac{4}{3} \cdot \frac{H^{2}}{b l}=1-\frac{150}{300}-\frac{150}{350}+\frac{4}{3} \cdot \frac{150^{2}}{300 \cdot 350}=\frac{5}{14}
$$

From eqn (33) 


$$
X=\frac{b-H}{2}=\frac{300-150}{2}=75 \mathrm{~mm}
$$

For masonry construction, the angle $\alpha \approx 45^{\circ}$, then according to eqn (32) the spall size coefficient

$$
n=\frac{X}{h}+\tan \alpha=\frac{0.075}{0.8}+1=1.1
$$

We can now determine the required masonry wall thickness for the guaranteed breach by a $25 \mathrm{~kg}$ TNT contact charge as

$$
h_{\text {req }}=\sqrt[3]{\frac{C \mu}{K_{0} n^{2}}}=\sqrt[3]{\frac{5 / 14 \cdot 25}{9.6 \cdot 1.1^{2}}}=0.92 \mathrm{~m}>h=0.8 \mathrm{~m}
$$

Since $h_{r e q}>h$, the masonry wall will be breached by the selected contact charge.

2) Using Table 4, the specific charge weight for forming an ejection crater can be taken as $K=2.0 \mathrm{~kg} / \mathrm{m}^{3}$ for masonry construction. Radius of the ejection crater can be determined from eqn (34) as

$$
r_{c}=\sqrt[3]{\frac{C}{18 K}}=\sqrt[3]{\frac{25}{18 \cdot 2}}=0.886 \mathrm{~m}
$$

Hence, the contact charge $C=25 \mathrm{~kg}$ TNT in the standard box will produce a breach hole in the $0.8 \mathrm{~m}$ thick masonry wall with a maximum diameter of the ejection crater on the front face

$$
D_{1}=2 \cdot 0.886=1.77 \mathrm{~m}
$$

Depth of the ejection crater can be determined approximately as

$$
P=(0.15 \ldots 0.2) r_{c}=(0.15 \ldots 0.2) \cdot 0.886=0.13 \ldots 0.18 \mathrm{~m}
$$

where the coefficient $0.15 \ldots 0.2$ is determined empirically for masonry and concrete walls.

Finally, a diameter of the spalling crater is given by

$$
D=2 n h=2 \cdot 1.1 \cdot 0.8=1.76 \mathrm{~m} \approx 1.8 \mathrm{~m}
$$

3) Graphical representation of the wall destruction and breaching process is shown in Figure 8.

\section{CONCLUSION}

In this paper, an analytical model of explosive breaching of walls with contact charges has been developed. The model provides a means for predicting the breach hole and crater dimensions and to construct the process of wall breaching graphically. Future efforts will address the calibration of the proposed model against the experimental data from the explosive tests on reinforced concrete and masonry wall targets to better understand the predictive capabilities of the proposed model. 


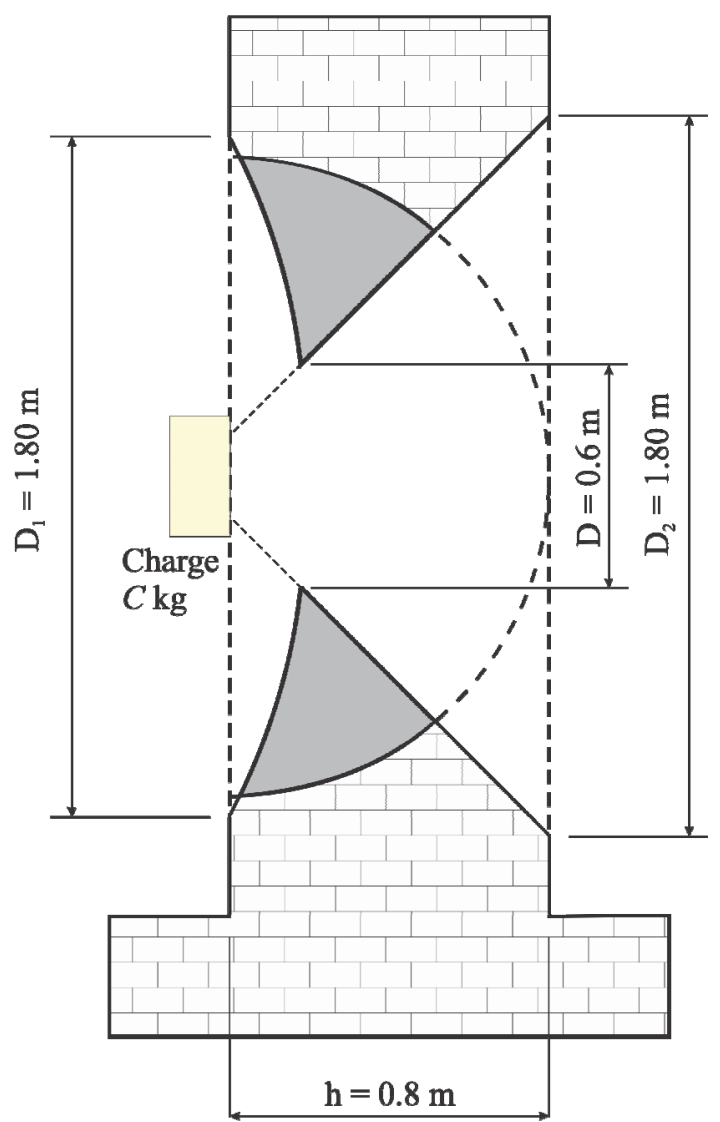

Figure 8. Mechanism of breaching of masonry wall with contact charge

\section{REFERENCES}

[1] Fujikake, K. and Aemlaor, P., Damage of reinforced concrete columns under demolition blasting, Engineering Structures, Vol. 55, 2013, pp. 116-125.

[2] Akers, S.A., Rickman, D.D., Ehrgott, J.Q. and Shelton, T.W., Explosive breaching of reinforced concrete walls: experimental efforts and numerical simulations, American Concrete Institute Special Publication SP 281-14, 2011, pp. 1-14.

[3] McVay M. K., Spall damage of concrete structures, Technical Report SL88-22, Department of the Army, Waterways Experimental Station, U.S. Corps Army of Engineers, 1988.

[4] Morishita M, Tanaka H, Ito M, Yamaguchi H., Damage of reinforced concrete slabs subjected to contact detonations, Journal of Structural Engineering, JSCE, 2000, Vol. 46A, 1787-1797 [in Japanese].

[5] Morishita M, Tanaka H, Ando T, Hagiya H., Effects of concrete strength and reinforcing clear distance on the damage of reinforced concrete slabs subjected to contact detonations, Concrete Research and Technology, 2004, 15(2), 89-98 [in Japanese].

[6] Beppu, M., Ohno, T., Ohkubo, K., Li, B., Satoh, K., Contact explosion resistance of concrete plates externally strengthened with FRP laminates. International Journal of Protective Structures, Vol. 1, No. 2, 2010. 
[7] Riedel, W., Mayrhofer, C., Thoma, K., Stolz, A., Engineering and numerical tools for explosion protection of reinforced concrete. International Journal of Protective Structures, Vol. 1, No. 1, 2010.

[8] Gebbeken, N., Greulich, S., Pietzsch, A., Landmann, F., The engineering-tool XploSim to determine the effects of explosive loadings on reinforced and fibre reinforced concrete structures, Proc. of 18th Int. Symp. Military Aspects of Blast and Shock, 2004, CD-ROM.

[9] FM 3-34.214. Explosives and demolitions, Department of the Army, Washington, DC, 2007.

[10] Krivtsun, V.I and Mentus, I.E., Determination of the basic characteristics of contact charges for breaching walls, Annual Publications of the Taras Shevchenko National University of Kiev, Ukraine, pp. 8-10, 2009 (in Ukrainian).

[11] Salamakhin, T. M., Fundamentals of mechanical effects of explosion on structures and methods of predicting blast loads, Moscow, Military Engineering Academy Publication (in Russian), 1974. 
